\title{
IS THE PEDESTRIAN AN ENDAGERED GROUP OF ROAD USERS? A DESCRIPTIVE ACCOUNT OF PEDESTRIAN INJURIES
}

\begin{abstract}
J. W. M. Kigera, $M B C h B$, Resident, Department of Orthopaedics, Makerere University, Kampala, Uganda and E.K.Naddumba, MBChB,MMed,FCS (ECSA), Senior Consultant Orthopaedic Surgeon, Department of Orthopaedics, Mulago Hospital, Kampala, Uganda
\end{abstract}

\section{ABSTRACT}

Background: Trauma is a leading cause of morbidity and mortality in Africa. Road traffic crashes are causative in majority of these cases. Pedestrians are vulnerable to injury in all types of collisions. This paper examines the injuries sustained by pedestrians.

Design: Prospective study.

Setting: Mulago Hospital, the National Referral Hospital in Uganda.

Patients and Methods: We prospectively recruited all musculoskeletal trauma patients who presented between August and December 2009 to casualty and the emergency ward and identified the patient characteristics and the nature of injuries sustained.

Results: Road Traffic Crashes (RTC's) were the leading cause of trauma and pedestrians were involved in $\mathbf{4 0 \%}$ of all RTC's. Motor vehicles and motorcycles were responsible for almost equal number of cases involving pedestrians. Pedestrians were younger than other R TC victims and sustained majority of the injuries over the weekend. The lower limbs were the commonest site of injury with fractures of the tibia and fibula being the commonest injury.

Conclusions: Pedestrians are vulnerable to injury in road traffic crashes and are bearing the brunt of these injuries. There is need to educate pedestrians on essentials of safety on the roads. Motorists and cyclists should also be sensitised on respect for other road users. There is a need for pedestrian walkways to reduce pedestrian injuries.

\section{INTRODUCTION}

Globally, trauma resulting from road traffic crashes is a major cause of death and disability with majority occurring in developing countries $(1,2)$. Pedestrians in the African Region are a vulnerable group with more than half of all RTC fatalities occurring among pedestrians (3). Pedestrian deaths are also involved in $45 \%$ of all RTCs deaths in low Income countries. It is estimated that over 230,000 pedestrians die in low income countries each year (3). Road Traffic Crashes have been on the rise in East Africa as shown in a study in Kenya. In urban areas the most vulnerable group are pedestrians (4). We conducted a prospective study to document the injuries sustained by pedestrians. We hypothesised that pedestrians were more likely to suffer lower limb injuries and were more likely to get injured in the hours of darkness and over the weekend.

\section{MATERIALS AND METHODS}

This prospective study included all musculoskeletal trauma patients who presented between August and December 2009 to the Accident and Emergency Department. All patients seen as outpatients or inpatients in the Accident and Emergency with musculoskeletal injuries were included. Patients with fractures and dislocations of the extremities and spine injuries were included. We excluded patients with isolated trauma to the head, chest and abdomen. We also excluded patients with burns and pathological fractures and soft tissue injuries to the extremities. Patient demographic data were obtained by interviewing the patients while injury details were obtained from the case notes of the admitting doctor and review notes of the resident on call. The lower limb included the pelvis and hip while the upper limb included the pectoral girdle. The data was collected by means of a questionnaire designed for that purpose.

Patients for whom there was incomplete data were excluded from the analysis. The study was conducted in Mulago Hospital, the National Referral Hospital in Uganda whose characteristics have been previously described (5). The study was approved by the institution review board. Data was entered in MS Excel and later exported into SPSS for windows version 11.5 (SPSS Inc., Chicago, Illinois). Statistical analysis was done using the Student $T$ test for continuous variables and the chi square for categorical variables.

\section{RESULTS}

Road Traffic Crashes (RTC'S) were the leading cause of trauma (55\%) with falls being the second leading cause of injury (28\%). Among the RTC's pedestrians were involved in $38 \%$ of all incidents. Motor vehicles 
caused $51 \%$ of all pedestrian injuries with motorcycles coming a close second with about $47 \%$. The rest of the injuries were caused by bicycles.

Males constituted $79.2 \%$ of the injured pedestrians with a male: female of about 4: I. The age histogram revealed a right sided skewed distribution and mean age was 27 years (Figure 1).The mean age of other RTC victims was 31.27 years and the difference in mean age was significant $(p=0.03)$.

Figure 1

Age distribution of patients

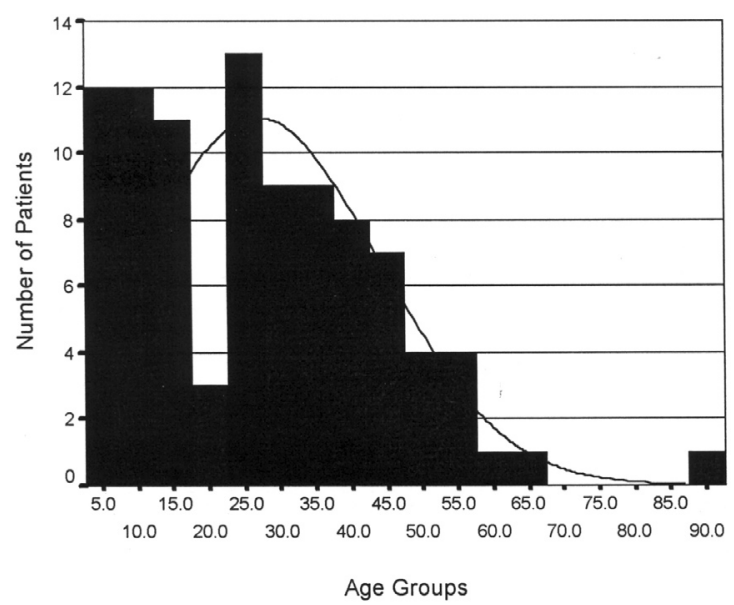

Majority of the patients (45.8\%) had achieved primary level of education with only $2.1 \%$ having university education (Table 1). Students comprised $36.2 \%$ of all patients with professionals being only $6.4 \%$ (Table 2).

Table 1

Education Level of Patients

\begin{tabular}{lcc}
\hline Category & Number & Percentage \\
\hline None & 8 & 8.5 \\
Pre Primary & 10 & 10.6 \\
Primary & 44 & 46.8 \\
Secondary & 24 & 25.5 \\
Post Secondary Training & 6 & 6.4 \\
University & 2 & 2.1 \\
\hline Total & 94 & 100 \\
\hline
\end{tabular}

Table 2

Occupation of the patients

\begin{tabular}{lcc} 
Category & Frequency & Percentage \\
\hline Student & 34 & 36.2 \\
Semi skilled & 25 & 26.6 \\
Unskilled & 22 & 33.4 \\
Professional & 6 & 6.4 \\
Housewife & 4 & 4.3 \\
Other & 3 & 3.2 \\
\hline Total & 94 & 100.0 \\
\hline
\end{tabular}

Majority of the injuries (54.2\%) occurred in the three days of the weekend with $45.8 \%$ of the injuries occurring between Monday and Thursdays. The time of injury -recorded showed that $39.56 \%$ of injuries occurred between midday and $6 \mathrm{pm}$ with only $4.4 \%$ of injuries occurring between midnight and 6 am (Table 3).

Table 3

Time of injury

\begin{tabular}{lcc}
\hline Time Period & Number & Percentage \\
\hline 0601 hrs - 1200 hrs & 24 & 26.37 \\
1201 hrs to $1800 \mathrm{hrs}$ & 36 & 39.56 \\
$1801 \mathrm{hrs}-0000 \mathrm{hrs}$ & 27 & 29.67 \\
$0001 \mathrm{hrs}-0600 \mathrm{hrs}$ & 4 & 4.40 \\
\hline
\end{tabular}

Majority of the patients (87.9\%) had isolated musculoskeletal injuries with $12.1 \%$ of patients having multiple site injuries. Of all the musculoskeletal injuries $80 \%$ were in the lower limbs $19 \%$ in the upper limbs and $1 \%$ in the spine. The majority of all injuries were to the leg causing fractures of the tibia and fibula (Table 4).

Table 4

Injuries sustained

\begin{tabular}{lcc}
\hline Site & Number & Percentage \\
\hline Tibia and fibula & 37 & 40.66 \\
Femur & 18 & 19.78 \\
Ankle & 10 & 10.99 \\
Radius ulna & 8 & 8.79 \\
Humerus & 7 & 7.69 \\
Foot & 4 & 4.40 \\
Clavicle & 2 & 2.20 \\
Pelvis & 2 & 2.20 \\
Elbow dislocation & 1 & 1.10 \\
Hip dislocation & 1 & 1.10 \\
Spine & 1 & 1.10 \\
\hline
\end{tabular}

\section{DISCUSSION}

Road traffic crashes are responsible for more than half of all trauma and pedestrians are involved in $38 \%$ of all RTCs. The majority of the patients were male with a mean age of 27 years. The average age of pedestrians was significantly different from other R TC patients.

Students constituted $36.2 \%$ of all patients. Majority of the injuries occurred over the weekend and the lower limbs were most commonly injured. The high numbers of pedestrian injuries has been shown in other urban areas in developing nations. It is also shown that majority of the injuries among school going children occur as they cross the road or playing (6). Although the circumstances surrounding the injuries in our study were not recorded, the high number of students may indicate that the mechanisms are similar to those in 
India. A study done in Kampala indicated that students traveling on their way to school were especially prone to pedestrian injuries (7). A study of RTC fatalities in Mexico showed that there was an increased incidence in patients over 55 years(8). This is in contrast to what we found and what was found by several other authors using fatalities, community or hospital data (9-12). The average age of male pedestrians injured was significantly lower than that of female pedestrians in a study in South Africa that collected data from adults above the age of 15 years (13). While we found that motorcycles were responsible for $47 \%$ of all injuries the situation is different in the west. A study of fatalities in the US found that motorcycles were responsible for a negligible proportion of pedestrian fatalities (9). In urban Kampala there is also a large number of injuries involving bicycles although the majority of these are not serious enough to warrant medical attention (7). In parts of Nigeria, more pedestrians were injured by motor vehicles than motorcycles (12) while in other parts of Nigeria motorcycles were the commonestcause of pedestrian injuries (14). In Thailand, motorcycles are responsible for a majority of pedestrian injuries (15). This is an indication of the differences in the types of vehicle on the roads in the two different regions. Studies in Nigeria have shown the vulnerability of pedestrians to trauma of the extremities when injured by a motorcycle $(10,16)$. This is similar to what we found in our study. The lower limbs are also prone to injury in crashes involving motor vehicles as shown in a study done in the US (11).

Though we did not collect data on injury severity or procedures done, the high morbidity of musculoskeletal injuries in pedestrians is evidenced by the fact that they frequently require operative procedures (11). It has been suggested that human error is the cause of most pedestrian injuries (4). This provides an avenue for possible remedial measures including enforcing the traffic laws and reducing drank driving. This may lead to a reduction of pedestrian injuries.

\section{CONCLUSIONS}

Pedestrians are vulnerable to injury in road traffic crashes and are bearing the brunt of these injuries. There is need to educate pedestrians especially school going children on essentials of safety on the roads. Motorists and cyclists should also be sensitised on respect for other road users. The law should prescribe stiff penalties for those causing pedestrian injuries. There is a need to create special lanes for pedestrian use only as this will reduce the human vehicular interaction that inevitably leads to pedestrian injury and death.

\section{REFERENCES}

1. Krug, E. Injury: a leading cause of global burden of disease. WHO: 1999.

2. WHO. World report on road traffic injury prevention: 2004.

3. Naci, H., Chisholm, O. and Baker,T.O.Distribution of road traffic deaths by road user group: a global comparison. Inj. Prev. 2009; 15(1): 55-59.

4. Odero,W., Khayesi, M. and Heda,P.M. Road traffic injuries in Kenya: Magnitude, causes and status of intervention. Injury Control and Safety Promotion. 2003; 10(1): 53 - 61.

5. Kigera, J.W. M. and Naddumba, E. K. Patterns of injuries after road traffic crashes involving Bodabodas. Ann. Afr. Surg. 2010; 5:12-15.

6. Poudel-Tandukar, K., Nakahara, S., Ichikawa, M., Poudel, K.C. and Wakai, S. Relationship between mechanisms and activities at the time of pedestrian injury and activity limitation among school adolescents in Kathmandu, Nepal. Accid Anal Prev. 2006; 38(6): 10581063.

7. Nakitto,M.T.,Mutto,M.,Howard, A.and Lett, R.Pedestrian traffic injuries among school children in Kawempe, Uganda. Afr. HIth. Sci. 2008; 8(3): 156-159.

8. HA-jar, M., Vazquez-Vela, E. and Arreola-Risa, C. Pedestrian traffic injuries in Mexico: A country update. Injury Control and Safety Promotion. 2003; 10(1): 37-43.

9. Paulozzi, L.J. United States pedestrian fatality rates by vehicle type. Inj Prev. 2005; 11(4): 232-236.

10. Solagberu, B.A., Ofoegbu, C.K. Nasir, A.A., Ogundipe, O.K., Adekanye, A.O. and AbdurRahman, L.O.Motorcycle injuries in a developing country and the vulnerability of riders, passengers, and pedestrians. Inj Prevo. 2006; 12(4): 266-268.

11. Peng, R.Y. and Bongard, F.S. Pedestrian versus motor vehicie accidents: an analysis of 5,000 patients. J. Am. Coll. Surg. 1999; 189(4): 343-348.

12. Ifesanya,A.O.,Afuwape,D.,Okoje,V.N.,etal.Unintentional injury outcomes secondary to pedestrian traffic crashes: a descriptive analysis from a major medical center. Prehosp. Dis. Med. 2009; 24(5): 443-446.

13. Hobday, M.B. and Knight, S. Motor vehicle collisions involving adult pedestrians in eThekwini in 2007. Intern. J. Injury Control Safety Promotion. 2010; 17 (1): 61-68.

14. Umaru, H., Ahidjo, A. and Dogo, H. Pedestrian injuries resulting from road traffic accidents: the Azare experience. Niger J.Med. 2007; 16(2): 169-172.

15. Swaddiwudhipong, W., Nguntra, P., Mahasakpan, P., Koonchote, S. and Tantriratna, G. Epidemiologic characteristics of drivers. vehicles pedestrians and road environments involved in road traffic injuries in rural Thailand. Southeast Asian J.Trop. Med. Pub. Health. 1994; 25(1): 37-44.

16. Oluwadiya, K.S., Oginni, L.M., Olasinde, A.A. and Fadiora, S.O. Motorcycle limb injuries in a developing country. West Afr. J. Med. 2004; 23(1): 42-47. 